\title{
Elevation in liver enzymes is associated with increased IL-2 and predicts severe outcomes in clinically apparent dengue virus infection
}

\author{
Thamarasi Senaratne ${ }^{1}$, Jillian Carr ${ }^{2}$ and Faseeha Noordeen ${ }^{1^{*}}$ \\ 1. Department of Microbiology, Faculty of Medicine, University of Peradeniya, Sri Lanka \\ 2. Microbiology and Infectious Diseases, \\ School of Medicine, Flinders University, South Australia
}

Corresponding Author

${ }^{1 *}$ Dr. F. Noordeen, Department of Microbiology, Faculty of Medicine, University of Peradeniya, Sri Lanka.

E mail: faseehan@pdn.ac.lk; faseeha.noordeen12@gmail.com

Telephone: +94812396532

\section{List of abbreviations \\ DENV - Dengue virus \\ DF - Dengue fever \\ DHF - Dengue haemorraghic fever \\ PCV - Packed cell volume \\ DSS - Dengue shock syndrome \\ WBC - White blood cell count \\ SEM - Standard error of mean \\ $\mathrm{CP} \quad$-Central Province \\ AST - Aspartate aminotransferase \\ ALT - Alanine Aminotransferase}

Keywords: Dengue fever, dengue haemorrhagic fever, TNF- $\alpha$ and IL-2 levels. 


\begin{abstract}
The objective of the present study was to assess the circulating TNF- $\alpha$ and IL-2 levels in dengue virus (DENV) infected patients and to correlate these with clinical severity of DENV infections. A single analyte quantitative immunoassay was used to detect TNF- $\alpha$ and IL-2 in 24 dengue fever (DF) and 43 dengue haemorraghic fever (DHF) patients, 15 healthy adults and 6 typhoid patients. The mean TNF- $\alpha$ and IL-2 levels of DENV- infected patients were higher than that of healthy adults and typhoid patients. No significant difference in TNF- $\alpha$ levels was noted between DFand DHF patients $(\mathrm{p}=0.5)$ but a significant increase in IL-2 levels was observed in DHF compared with DF patients (mean of $\mathrm{DF}=59.7 \mathrm{pg} / \mathrm{mL}$, mean of $\mathrm{DHF}=166.9 \mathrm{pg} / \mathrm{mL} ; p=0.02$ ). No significant association of TNF- $\alpha$ or IL-2 levels was noted with packed cell volume (>45), thrombocytopenia, leucopenia or the presence of viraemia. The liver function tests measuring AST (aspartate aminotransferase) $(p=0.01)$ and ALT (alanine aminotransferase) $(p=0.02)$ levels were significantly elevated in DENV-infected patients. AST:ALT was significantly elevated in DHF/DSS (Dengue shock syndrome) compared with DF patients. A significant positive linear correlation was noted between AST and IL-2 $(r=0.31 ; p=0.01)$ and ALT and IL-2 elevations $(r=0.2 ; p=0.02)$. Thus, AST and ALT levels correlate with both disease severity and circulating IL-2 levels. We suggest a role for circulating IL-2 in liver dysfunction and propose that a combined assessment of AST/ALT in conjunction with IL-2 at the early stages of symptomatic DENV infection may be useful to predict the pression to severe forms of dengue.
\end{abstract}




\section{Introduction}

Clinical presentation of DENV infection varies from a mild self-limiting DF and more severe forms such as DHF and dengue shock syndrome (DSS). Pathogenesis of more severe forms of DENV infection is believed to be due to host genetic factors, host immunity, including the release of pro-inflammatory and vasoactive cytokines, chemokines and the virulence of different DENV strains [1-3].The more severe form of DENV infection (DHF and DSS) present later in infection at the time of defervescence and decline in viraemia [4]. Only a small percentage of the many DENV infected cases will develop severe disease [4]. Many studies have found differences between DF and DHF with regard to T-cell responses and support considering DF and DHF as separate clinical entities. However, according to the latest WHO guidelines, DF and DHF have diverse clinical presentations and not all the severe DHF cases lead to shock or DSS [5]. Hence, predicting the likelihood of development of DHF and DSS is a major issue for patient management. Therefore, defining the progression of the clinical DENV infection and associated biological markers at an early stage that are later associated with severe infection outcomes will facilitate a better prognosis for DHF and DSS. This would have clear opportunities for improvements in the management of DENV-infected patients, particularly in times of outbreak in resource poor settings, such as has occurred recently in the Central Province of Sri Lanka [6].

Both innate and adaptive or cell mediated immune responses are known to contribute to the progression of DENV infection. When a DENV infection progresses in a human host, cytokines are secreted mainly by monocytes, spleenic macrophages and B and T lymphocytes $[7,8]$. According to Chaturvedi et al, high levels of tumour necrosis factor alpha (TNF- $\alpha$ ), interleukin-2 (IL-2), interleukin-6 (IL-6) and interferon alpha (IFN- $\alpha$ ) have been detected in the serum in the 
first three days of DF whereas interleukin-10 (IL-10), interleukin-5 (IL-5) and interleukin-4 (IL4) are seen in the latter phase of DF, after 4 days of fever [3]. Increased pro-inflammatory cytokines, including TNF- $\alpha$ are found to be in higher concentration in the circulation of DHF patients than in DF patients and healthy controls in a number of studies [9-13]. Moreover, studies using murine models have shown high DENV titre, macrophage infiltration and TNF- $\alpha$ production to be the main events leading to haemorrhage [14-16]. Thus, elevated levels of TNF$\alpha$ have been strongly linked to dengue severity in both human and mouse studies.

IL-2 is a major driver of immune responses and has also been detected at elevated levels in DF/DHF patients [3,13]. A higher level of IL-2 in DHF patients has been suggested to cause plasma leakage and shock in DSS [13]. IL-2 is well described to be an important factor in T-cell growth, differentiation and T-cell responses. The role of T-cell responses in the progression of DENV infection into severe disease is not understood completely [17]. Additionally, it is thought that there may also be differences in the T-cell response between primary and secondary DENV infections [13]. Several studies done in dengue endemic countries have shown that DENV specific T-cell responses contribute to the protection against infection [18]. Hence, T-cell responses are linked to dengue disease and changes that lead to alterations in T-cell responses, such as IL-2 levels, may slow the process of viral clearance, or lead to over activation of T-cells, and may influence the severity of disease [19].

Studies done in several countries, such as Thailand and Singapore have established a correlation between the TNF- $\alpha$ and IL-2 response with disease severity $[11,2,20]$. Very few studies, however have undertaken analysis of cytokine levels during DENV infection using the Sri Lankan 
population. One study done in Colombo, the Western Province of Sri Lanka failed to find any association between the leukocyte counts and TNF- $\alpha$ levels or any association between TNF- $\alpha$ levels with the primary or secondary dengue[21]. Further, in Sri Lanka, the incidence of DF and DHF is increasing and we have observed a significant number of severe disease associated with primary DENV infection (Senaratne et al unpublished data). This changing disease profile and known contrasts to the prior literature from other countries justifies specific analysis of factors such as TNF- $\alpha$ and IL-2 in the Sri Lankan population.

Hence, we investigated the role of IL-2 and TNF- $\alpha$ in the pathogenesis of acute DENV infection and correlated circulating levels of IL-2 and TNF- $\alpha$ with varying clinical severity of DENV infection. Our data demonstrate significant association between circulating levels of IL-2 and TNF- $\alpha$ with changes in liver enzymes at an early stage of the clinical disease is predictive of severe forms of DENV infections. Our study herein is one of the first studies using a cohort of DENV infected patients from Sri Lanka, where dengue has evolved to become the number one vector born insult to public health, to quantitate TNF- $\alpha$ and IL-2 levels together with serological and molecular data. This study will also significantly advance our understanding of the changing incidence and disease presentation of DENV infection in the dengue endemic region.

\section{Materials and methods}

\subsection{Study population}

This is a cross sectional prospective study carried out from July 2011 to February 2012 using patients ( $\mathrm{n}=292)$ admitted to the General Hospital Kandy (GHK), Sri Lanka. Ethical clearance 
was obtained from Ethical Review Committees of Faculty of Medicine, University of Peradeniya (2011/EC/08) and GHK, Sri Lanka.

\subsection{Clinical data}

Informed consent was obtained and all the clinical data was collected using a detailed questionnaire, which included the data on patient details, presenting illnesses, clinical data, dengue specific data (anti-DENV IgM/IgG detection and nucleic acid detection) and non-specific laboratory data (haemataological parameters and liver enzymes activity).

Five $\mathrm{mL}$ of blood was collected from clinically suspected DF/DHF patients presenting to hospital on fever days 5 or less (onset of fever was considered as day 1). The classification of disease grade was done by the attending physician according to the WHO DF/DHF classification [5]. Presenting illnesses were recorded as fever, history of fever, headache, retro orbital pain, myalgia, arthralgia or rash. DF was classified as any acute febrile illness with one of the following: myalgia, headache, retro-orbital pain, bleeding, shock or low platelet count. DHF was classified as any haemorrhagic manifestations such as positive tourniquet test (PTT), presence of petechiae, ecchymosis or purpura, bleeding in the mucosa, gastro intestinal tract and cannula site. Leakage was recorded as pleural effusion, ascites, liver enlargement or shock.

Haematological laboratory investigations carried out in these patients included total white blood cell count (WBC), platelet counts, haemoglobin, and packed cell volume (PCV) levels. These data were recorded on admission and whenever available in the latter part of the disease. Initial clinical diagnosis on admission and the final diagnoses were also recorded. 
RT-PCR was performed on all patients' samples using DENV specific primers (DV1 and DV3) for the envelope gene and (D1 and D2) for the capsid gene detection followed by DENV typing with serotype specific primers, as previously described [22,23].Anti-DENV IgM detection was performed for patients by a commercial ELISA in accordance with manufacturer's instructions (Standard Diagnostics, Korea). A total of 67 DENV-infected patients either confirmed by serological assays or RT-PCR were selected for the cytokine analysis (DF, n=24 and DHF, $\mathrm{n}=43)$.

\subsection{Cytokine assays}

Quantitation of two cytokines, TNF- $\alpha$ and IL-2 in the plasma samples was performed using the commercially available cytokine ELISA (Single analyte ELISArray-Qiagen, Germany) for the 67 confirmed dengue cases, 15 healthy age matched and non febrile controls and 6 blood culture confirmed typhoid cases. The typhoid cases were included in this study as a febrile comparison and in order to define the different changes in the cytokine levels in typhoid, another confounding presenting febrile illness in Sri Lanka compared with DENV infection. No published data were available on the IL-2 and TNF- $\alpha$ levels in the Sri Lankan typhoid patients for the comparison. All these samples were tested in duplicates. Internal quality control standards were included in each assay and the standard curve was used for the estimation of cytokine concentration $(\mathrm{pg} / \mathrm{mL})$. 


\subsection{Statistical analysis}

Statistical analysis was done using Minitab, Version 17.0 and graphs were developed using Graph Pad Prism, Version 6.0 (San Diego, California). The comparison between groups was expressed as mean with standard error of mean (SEM) values. Mann-Whitney U test (two tailed) was used to find the differences in mean cytokine levels in different groups. Tukey test was performed to find out the difference within the groups. Pearson's correlation was performed to find out the negative and positive correlation between cytokine levels and varying clinical severity. Spearson's correlation was performed to assess the differences in the cytokine profiles within each serotype of DENV-infected patients. A statistical test with $p$-value $<0.05$ was considered significant in the mean comparison of variables or in the correlation analysis.

\section{Results}

\subsection{The clinical profile and viral characteristics of dengue patient and control cohorts}

Of the 67 DENV-infected patients selected for cytokine analysis, 48 (71.7\%) were males and 19 (28.31\%) were females. Our healthy cohort was $66.7 \%$ male, $33.3 \%$ female, and similarly typhoid comparison cohort was also $66.7 \%$ male and $33.3 \%$ female. The mean age of the DENV cohort was $28.07(\mathrm{SD}=12.89)$ with a mean duration of fever 4.37 days. The mean age of the afebrile healthy control cohort was $34.13(\mathrm{SD}=12.64)$ while the mean age of the typhoid cohort was $11.16(\mathrm{SD}=6.88)$ with a mean duration of fever 10.7 days. In the dengue cohort, fifteen patients (22.28\%) showed bleeding manifestations while 28 (41.79\%) showed liver enlargement. Neither bleeding manifestations nor liver enlargement was present in the healthy or typhoid control cohorts. Twenty four (35.8\%) of DENV-infected patients were clinically classified as DF and $43(64.2 \%)$ as DHF. Of the 43 patients with DHF, 21/43 (49\%) patients had a primary 
DENV infection and 22/43(51\%) had a secondary DENV infection, as indicated by the presence of anti-DENV IgM and $\operatorname{IgG}$ at presentation. There was a significant reduction in the platelet counts of DHF patients (mean \pm SD- $145.2 \times 10^{9} / \mathrm{L} \pm 39.5 \times 10^{9} / \mathrm{L}$ ), compared to the DF patients $\left(\right.$ mean \pm SD-93.7 $\left.\times 10^{9} / \mathrm{L} \pm 52.3 \times 10^{9} / \mathrm{L}\right) \quad(p<0.05)$. Platelet counts were not determined in the healthy cohort, or in the typhoid control group.

\subsection{TNF- $\alpha$ and IL-2 are elevated in DENV-infected patients, with increased IL-2 associated with severity of DENV infection}

We next measured and compared the levels of TNF- $\alpha$ and IL-2 in DENV-infected patients, grouped as either DF or DHF, with healthy controls and typhoid patients. The mean values and range of TNF- $\alpha$ and IL-2 concentrations varied among the different groups and are shown in Table 1 and graphically in Figure 1.Levels of TNF- $\alpha$ were significantly increased in DENVinfected patients compared to healthy controls $(p=0.005)$, although there was no significant difference in TNF- $\alpha$ levels in DF compared with DHF patients $(p=0.47)$ (Table 1, Figure 1A). Similarly, levels of IL-2 were significantly increased in DENV-infected patients $(p=0.008)$ but in this instance, IL-2 was also significantly elevated in DHF compared with DF patients $(p=0.02)$ (Table 1, Figure 1B). In comparison to typhoid patients, there was no significant difference in TNF- $\alpha$ or IL-2 levels when dengue was considered as a whole. In comparison, however, of typhoid patients with DHF, IL-2 levels were significantly elevated (Table 1, Figure 1B).

Further, Spearman's ranking test was performed to assess the correlation of TNF- $\alpha$ or IL-2 levels with the varying degrees of DHF severity. TNF- $\alpha$ showed no correlation with the disease severity $(r=0.18, p=0.15)$ whereas IL-2 showed a significant positive correlation with the disease severity $(r=0.38, p=0.002)$. 
Significant association was noted between each cytokine level with age (TNF- $\alpha$ and IL-2, $p=0.01)$ but no significant association was noted between either cytokine level with the time post onset of fever (Table 2 and Figure 2).

We next assessed the correlation of TNF- $\alpha$ or IL-2 level with classification of DENV infection as primary or secondary. No significant association was noted between primary or secondary DENV infections or with the anti-DENV IgM or IgG positivity (Table 2) and no correlation was noted between each cytokine in primary or secondary DENV infections (Table 3).

We then assessed the correlations of TNF- $\alpha$ or IL-2 level with various clinical presentations. However, a significant positive correlation was noted only between IL-2 level and arthralgia (Table 3). Of the laboratory data collected, a significant correlation was noted only between clinical leucopoenia with the level of TNF- $\alpha$ (Table 2). However, IL-2 showed a significant positive correlation with total WBC count (Table 3). Since platelet counts were similarly discriminated between DF and DHF, we next correlated TNF- $\alpha$ and IL-2 levels with platelet counts in DENV-infected patients. However, no significant association was observed between TNF- $\alpha$ or IL-2 levels and platelet count.

We next assessed the correlation between each TNF- $\alpha$ or IL-2 level with different serotypes of DENV and again found no significant association with either parameter with a specific DENV infection (Table 3). 
Of the DENV-infected patients admitted to hospital, 47.76\% ( $n=32 / 67)$ showed a clinically significant rise in AST:ALT (>2U/L).The AST:ALT mean \pm SD in patients classified as DF was 1.64 $\pm 0.74 \mathrm{U} / \mathrm{L}$ compared with 3.18 $\pm 4.50 \mathrm{U} / \mathrm{L}$ among patients classified as DHF/DSS. However, this was not statistically significant $(p=0.10)$. No significant correlation could be built between the AST:ALT ratio with the age, gender or the days post onset of fever.

We also correlated circulating TNF- $\alpha$ and IL-2 levels with ALT and AST levels in these patients. No significant correlation was noted between the AST:ALT ratio and TNF- $\alpha$ or with IL-2. When AST and ALT levels were considered individually, a significant positive linear correlation was observed between AST and IL-2 levels $(r=0.31 p=0.01)$ (Figure 3A) and also between ALT and IL-2 levels $(r=0.27 p=0.02$ ) (Figure 3B). No significant correlation was noted between $\operatorname{AST}(r=0.01, p=0.93)$ or ALT $(r=-0.07, p=0.59)$ with TNF- $\alpha$.

\section{Discussion}

In the present study we have defined the clinical, virological and immunological profiles in patients with confirmed DENV infection from the Kandy District, Central Province (CP) of Sri Lanka and correlated these observations with circulating TNF- $\alpha$ and IL-2 levels. Around 2000 clinically suspected DF/DHF cases have been reported annually from the CP of Sri Lanka and our own observations have supported a growing incidence of disease in this region [6]. We found a marked cytokine response among DF/DHF patients when compared to healthy controls and typhoid patients with statistically significant elevated levels of TNF- $\alpha$ and IL-2 in DENV infected patients (Figure 1A and Figure 1C). However, no significant discrimination was noted in TNF- $\alpha$ level between DF and DHF patients (Figure 1B) but a significant variation was 
observed in the IL-2 levels with further elevation of IL-2 in DHF compared with DF patients (Figure 1D). No significant correlation was observed between TNF- $\alpha$ and IL-2, suggesting the independent control and actions of these cytokines during DENV infection.

Our study is in agreement with the findings of the study done by Priyadarshani et al (2010) which shows no significant difference in the TNF- $\alpha$ levels with the disease grade [24]. In contrast to our data, some published data suggests a correlation between increased TNF- $\alpha$ levels and haemorrhagic tendencies both in human studies and in mouse models [14,15]. A significant increase in TNF- $\alpha$ was noted in secondary DENV infected patients during the latter phase of DF/DHF (after fever days 5) in previous studies [2,25]. The discrepancy in the TNF- $\alpha$ level between DF/DHF patients in the current study and previously published studies may be due to the differences between infecting DENV serotypes and strains, the different time points analysed post-infection (Figure 2) or differences in the genetic polymorphisms in the TNF- $\alpha$ gene in our study compared with other study populations [26]. This highlights the need for local characterization of disease profiles, including associated biomarkers including cytokines. Our study only analysed < day 5 of the onset of symptoms/fever and it is possible our samples may have been collected before the critical elevation of TNF- $\alpha$ expression associated with DHF.

Our association of IL-2 with dengue disease severity is in contrast to the findings of Malavige et al (2004) in Sri Lanka and Chakravarti et al (2006) in India [25,27]. In our study, a significant variation was observed in the IL-2 levels between DF and DHF patients $(p=0.02)$. Moreover, another study has shown IL-2 levels to be higher in DHF cases [13] supporting the findings of our current study on elevated IL-2 levels in DHF patients. Interestingly, comparatively higher 
levels of IL-2 were shown on day 4 of the illness in our study (Figure 2). Induction of plasma leakage in pathogenic infections by IL-2 through altering endothelial permeability has been demonstrated in cell culture even though the exact mechanism is not well understood [28]. Our study also shows the association between DHF and IL-2 elevation. IL-2 might directly contribute to the leakage seen in DHF through altering endothelial permeability or indirectly via known effects of IL-2 on T-cell responses. Thus our data supports a role for circulating IL-2 as a potential driver of severe dengue disease. We observed a significant increase of both TNF- $\alpha$ and IL-2 levels with the advancement of age. Very little information is available on the association of the cytokine levels with the age. In contrast to our findings, Hober et al has found higher TNF- $\alpha$ levels in children with dengue whereas the increase was moderate in adults with dengue when compared to healthy individuals [9]. However, no literature is available on the association between the IL-2 levels and the age of the dengue patients to support or refute our findings.

In our study, TNF- $\alpha$ and IL-2 levels yielded a broad range and a wider SD for resultant values. This large variation was observed in both DENV-positive patients, and healthy controls (Table 1). This may reflect the variability of study population in the production of these cytokines and may have reduced our ability to detect less prominent correlations of various factors, with these cytokines, such as the associations of TNF- $\alpha$ with severity of disease, as described above. The DENV-induced changes in TNF- $\alpha$ and IL-2 were, however, still succinct enough to identify the novel correlations we have described above. This variation should be taken into account in future studies and the sample size adjusted accordingly to ensure appropriately powered investigations.

We also measured DENV RNA and did not observe a significant association in the TNF- $\alpha$ and IL-2 variations between the viraemic (PCR positive) and non-viraemic (PCR negative) DF/DHF 
patients. This suggests that the presence of detectable virus in the circulation has no effect on the TNF- $\alpha$ and IL-2 responses (Table 2). Some studies have noted different DENV serotypes/strains to be more virulent than others with regards to inducing the pro-inflammatory cytokine response $[29,30]$. Our study encompassed samples from different serotypes, although predominantly DENV-1 and some patients even with infections with multiple DENV serotypes (results not shown). However, no significant association was observed between TNF- $\alpha$ or IL-2 levels and DENV serotype in our study cohort.

No significant association was noted between the TNF- $\alpha$ and IL-2 levels and increase in PCV (>45), thrombocytopenia, or with any of the major presenting illnesses or symptoms of DENV infection. However, we noted a significant positive association between leucopoenia and TNF- $\alpha$ (Table 2) and a positive correlation between IL-2 response with arthralgia and WBC count on admission. No published literatures are available on these associations in other DENV-infected cohorts to support or oppose our findings. Studies on dengue have shown a positive correlation between thrombocytopenia with the increase in other pro-inflammatory cytokines such as interferon-alpha [1]. Interestingly, a study done in patients with Ewing's sarcoma has noted the presence of thrombocytopenia when recombinant TNF- $\alpha$ was administered [31]. Another study has found a weak correlation with the increase of PCV and increase in IFN- $\gamma$ response in DENV infected patients [32]. Thus, there is some evidence of association of thrombocytopenia with TNF- $\alpha$ levels and PCV with cytokine levels, but outcomes of our studies do not support a specific association of these parameters with TNF- $\alpha$ or IL-2 during DENV infection. 
Our study shows no correlation between anti-DENV $\operatorname{IgM} / \operatorname{IgG}$ status and thus primary or secondary DENV infection with TNF- $\alpha$ and IL-2 cytokine levels. This is in contrast to a previous study which suggested the deposition of anti-DENV IgM or IgG antibody immune complexes in the blood capillaries may cause endothelial damage through production of TNF- $\alpha$ [2] and thus, our study does not support this potential association and proposed mechansim of severe dengue.

During DENV infection, the changes in the liver enzymes such as AST and ALT levels differ when compared to variations shown in other viral infections such as viral hepatitis which cause liver damage [33]. In DF/DHF patients, levels of both enzymes are increased but in particular, AST levels increase more than ALT [34]. The underlying reason behind this elevation is not properly understood. Potential mechanisms include direct viral hepatotoxicity, indirect cytokine or chemokine mediated liver toxicity, or for the case of AST levels, potential release from other cellular sources such as erythrocytes or monocytes [33]. Taken together the liver enzymes profiles in our study, a positive correlation between the elevations of the liver enzymes with dengue disease severity [35] as well as positive correlations of elevated cytokines and dengue severity, suggests an association between the elevation of liver enzymes and cytokine levels indirectly. Previous studies have shown significant positive associations between several cytokines including IFN- $\gamma$, IL-6 and IL-8 with the ALT levels during DENV infection in an Indian cohort [24]. The majority of the patients with increased ALT levels also showed pleural effusion/ascites and were clinically classified as DHF/DSS [24]. However, limited studies have been conducted on the association of IL-2 and TNF- $\alpha$ with AST/ALT levels in DENV infected patients with disease severity. Almost half of our study population showed AST:ALT $>2$ indicating acute changes in liver function and the potential for liver derangement due to DENV 
infection. This is consistent with DENV infection as a major cause of acute liver disease and increased AST and ALT's reported in DENV infected patients in a number of other studies [3537]. Prior studies have shown that liver derangement during DENV infection often occurs later in infection [34]. In our study the majority of the DF cases showed statistically significant lower AST and ALT levels compared to the DHF cases. Additionally, there was a statistically significant positive correlation between the IL-2 with AST and ALT levels (Figure 3), although no correlation was noted between AST and ALT with TNF- $\alpha$. The underlying mechanism of acute liver dysfunction and the role of the host immune response in symptomatic DENV infection is not well explained [33]. Previous studies where recombinant IL-2 was administered to humans has demonstrated that this can result in hepatotoxicity [38] and thus there is a precedent for a direct role of IL-2 in inducing the observed changes in AST and ALT levels seen in our study. This, however, remains to be directly tested in DENV infection. Prior studies in a Malaysian cohort reported negative correlations of cytokines such as IL-4, IL-12 and IL-13 in the febrile phase of dengue with warning signs and AST measurements but no reported associations of TNF- $\alpha$ or IL-2 with AST or ALT [39]. The positive correlations between elevations of AST and ALT with IL-2 and the association of higher levels of these factors in DHF/DSS compared with DF is the first report of this association in the literature. The growing recognition of acute liver disease associated with DENV infection [35] and our association shown herein warrants further investigation of the role of IL-2 in liver dysfunction during the acute febrile phase of dengue. The current study findings thus suggest that these parameters may be useful predictors for the progression of DENV infection to severe forms of dengue at least in our Sri Lankan cohort. 
In summary, we have shown an association of the clinical profile of DF/DHF patients with TNF$\alpha$ and IL-2 levels in the circulation. While both TNF- $\alpha$ and IL-2 are elevated in DENV-infected patients, TNF- $\alpha$ shows no significant association with the disease severity (DF and DHF) whereas IL-2 shows a positive correlation with the clinical severity in DENV infected DF/DHF patients and is further associated with increased markers of liver dysfunction. Thus, our results suggest that higher levels of IL-2, AST and ALT, but not TNF- $\alpha$ at the early stages $(<5$ days post fever) of the disease may be useful as prognostic markers for the progression to severe forms of DENV infections, at least in our Sri Lankan patients, in whom severe forms of DENV infection have become more prevalent in recent years. We anticipate expanding these studies with larger sample size and samples collected at later time points of DF to further validate this association and define the roles and prognostic utility of IL-2 and liver function in severe forms of DF/DHF.

Competing interests: None declared.

\section{Authors' contributions}

TS carried out the samples collection, analysis, immunoassays and other laboratory experiments and drafted the manuscript. FN participated in the study design, helped to draft and improve the manuscript. JC critically read and contributed to improve the manuscript. All authors read and approved the final manuscript. 


\section{Acknowledgements}

This work was supported by National Science Foundation of Sri Lanka (NSF/SCH/12/03) and

National Research Council of Sri Lanka (NRC/RG/11/129). The authors acknowledge Ms.

Kiruthiha Ehambaram, Research Assistant, Department of Parasitology, Faculty of Medicine, University of Peradeniya for helping with statistical analysis. The authors also thank Ms. Thevaki Gnanakarunyan, Faculty of Medicine, University of Jaffna for providing blood samples of typhoid patients.

\section{FIGURE LEGENDS}

Figure1. Association between circulating cytokine levels and dengue severity. Blood was collected at initial hospital presentation from patients classified as DF, DHF/DSS or typhoid or from the healthy control cohort. Samples were analysed for A. TNF- $\alpha$ Vs healthy and DENVinfected patients (DF+DHF/DSS); B. TNF- $\alpha$ Vs stratified groups; C. IL-2 Vs healthy and DENV-infected patients (DF+DHF/DSS); D. IL-2 Vs stratified groups; * =significantly different compared to healthy controls as tested by ELISA (Mann-Whitney test).

Figure 2. Circulating cytokine levels in DENV-infected patients with time. Blood was collected from 3-5 days post onset of fever and analyzed for TNF- $\alpha$ and IL-2 by ELISA. No significant difference in TNF- $\alpha$ or IL-2 levels was observed with time (one way ANOVA).

Figure 3. Correlation of liver enzyme activity with circulating IL-2. Liver function tests (AST or ALT) were done on admission after clinical evaluation and correlated with the level of IL-2 
measured in the blood by ELISA. A. AST and IL-2 $(r=0.31, p=0.01)$; B. ALT and IL-2 $(r=0.27$, p=0.02) (Pearsons correlation).

\section{References}

[1] Tang Y, Kou Z, Zhang F, Yao X, Liu S, Ma J, et al. Both viremia and cytokine levels associate with the lack of severe disease in secondary dengue 1 infection among adult Chinese patients. PloS one. 2010;5:e15631.

[2] lyngkaran N, Yadav M, Sinniah M. Augmented inflammatory cytokines in primary dengue infection progressing to shock. Singapore medical journal. 1995;36:218-21.

[3] Chaturvedi UC, Agarwal R, Elbishbishi EA, Mustafa AS. Cytokine cascade in dengue hemorrhagic fever: implications for pathogenesis. FEMS immunology and medical microbiology. 2000;28:183-8. [4] Simmons CP, Farrar JJ, van Vinh Chau N, Wills B. Dengue. New England Journal of Medicine. 2012;366:1423-32.

[5] WHO. Comprehensive Guidelines for Prevention and Control of Dengue and Dengue Haemorrhagic Fever. world health organization; 2011. p. 78.

[6] Senaratne TN, Noordeen F. Diagnosis of dengue in Sri Lanka: improvements to the existing state of the art in the island. Transactions of the Royal Society of Tropical Medicine and Hygiene. 2014;108:68591.

[7] Rothman AL. Immunity to dengue virus: a tale of original antigenic sin and tropical cytokine storms. Nature reviews Immunology. 2011;11:532-43.

[8] Pawitan JA. Dengue virus infection: predictors for severe dengue. Acta medica Indonesiana. 2011;43:129-35.

[9] Hober D, Poli L, Roblin B, Gestas P, Chungue E, Granic G, et al. Serum levels of tumor necrosis factoralpha (TNF-alpha), interleukin-6 (IL-6), and interleukin-1 beta (IL-1 beta) in dengue-infected patients. The American journal of tropical medicine and hygiene. 1993;48:324-31.

[10] Kittigul L, Temprom W, Sujirarat D, Kittigul C. Determination of tumor necrosis factor-alpha levels in dengue virus infected patients by sensitive biotin-streptavidin enzyme-linked immunosorbent assay. Journal of virological methods. 2000;90:51-7.

[11] Green S, Vaughn DW, Kalayanarooj S, Nimmannitya S, Suntayakorn S, Nisalak A, et al. Early immune activation in acute dengue illness is related to development of plasma leakage and disease severity. The Journal of infectious diseases. 1999;179:755-62.

[12] Bethell DB, Flobbe K, Cao XT, Day NP, Pham TP, Buurman WA, et al. Pathophysiologic and prognostic role of cytokines in dengue hemorrhagic fever. The Journal of infectious diseases. 1998;177:778-82.

[13] Kurane I, Innis BL, Nimmannitya S, Nisalak A, Meager A, Janus J, et al. Activation of T lymphocytes in dengue virus infections. High levels of soluble interleukin 2 receptor, soluble CD4, soluble CD8, interleukin 2, and interferon-gamma in sera of children with dengue. The Journal of clinical investigation. 1991;88:1473-80.

[14] Yen YT, Chen HC, Lin YD, Shieh CC, Wu-Hsieh BA. Enhancement by tumor necrosis factor alpha of dengue virus-induced endothelial cell production of reactive nitrogen and oxygen species is key to hemorrhage development. Journal of virology. 2008;82:12312-24.

[15] Shresta S, Sharar KL, Prigozhin DM, Beatty PR, Harris E. Murine model for dengue virus-induced lethal disease with increased vascular permeability. Journal of virology. 2006;80:10208-17. 
[16] Chen HC, Hofman FM, Kung JT, Lin YD, Wu-Hsieh BA. Both virus and tumor necrosis factor alpha are critical for endothelium damage in a mouse model of dengue virus-induced hemorrhage. Journal of virology. 2007;81:5518-26.

[17] Midgley CM, Bajwa-Joseph M, Vasanawathana S, Limpitikul W, Wills B, Flanagan A, et al. An indepth analysis of original antigenic sin in dengue virus infection. Journal of virology. 2011;85:410-21. [18] Weiskopf D, Angelo MA, de Azeredo EL, Sidney J, Greenbaum JA, Fernando AN, et al.

Comprehensive analysis of dengue virus-specific responses supports an HLA-linked protective role for CD8+ T cells. Proceedings of the National Academy of Sciences of the United States of America. 2013;110:E2046-53.

[19] Malavige GN, Jeewandara C, Alles KML, Salimi M, Gomes L, Kamaladasa A, et al. Suppression of Virus Specific Immune Responses by IL-10 in Acute Dengue Infection. PLoS neglected tropical diseases. 2013;7:e2409.

[20] Libraty DH, Endy TP, Houng HS, Green S, Kalayanarooj S, Suntayakorn S, et al. Differing influences of virus burden and immune activation on disease severity in secondary dengue- 3 virus infections. The Journal of infectious diseases. 2002;185:1213-21.

[21] Malavige GN, Huang L-C, Salimi M, Gomes L, Jayaratne SD, Ogg GS. Cellular and Cytokine Correlates of Severe Dengue Infection. PloS one. 2012;7:e50387.

[22] Chow VT, Seah CL, Chan YC. Use of NS3 consensus primers for the polymerase chain reaction amplification and sequencing of dengue viruses and other flaviviruses. Archives of virology.

1993;133:157-70.

[23] Lanciotti RS, Calisher CH, Gubler DJ, Chang GJ, Vorndam AV. Rapid detection and typing of dengue viruses from clinical samples by using reverse transcriptase-polymerase chain reaction. Journal of clinical microbiology. 1992;30:545-51.

[24] Priyadarshini D, Gadia RR, Tripathy A, Gurukumar KR, Bhagat A, Patwardhan S, et al. Clinical findings and pro-inflammatory cytokines in dengue patients in Western India: a facility-based study. PloS one. 2010;5:e8709.

[25] Chakravarti A, Kumaria R. Circulating levels of tumour necrosis factor-alpha \& interferon-gamma in patients with dengue $\&$ dengue haemorrhagic fever during an outbreak. The Indian journal of medical research. 2006;123:25-30.

[26] Gupta R, Kapuria V, Das SN. Single nucleotide polymorphisms in TNF-alpha, TNFR2 gene and TNFalpha production in Asian Indians. Immunological investigations. 2009;38:240-54.

[27] Malavige GN, Fernando S, Fernando DJ, Seneviratne SL. Dengue viral infections. Postgraduate medical journal. 2004;80:588-601.

[28] Cotran RS. American Association of Pathologists president's address. New roles for the endothelium in inflammation and immunity. The American journal of pathology. 1987;129:407-13.

[29] Lanciotti RS, Gubler DJ, Trent DW. Molecular evolution and phylogeny of dengue-4 viruses. The Journal of general virology. 1997;78 ( Pt 9):2279-84.

[30] Chungue E, Cassar O, Drouet MT, Guzman MG, Laille M, Rosen L, et al. Molecular epidemiology of dengue-1 and dengue-4 viruses. The Journal of general virology. 1995;76 ( Pt 7):1877-84.

[31] Michelmann I, Bockmann D, Nurnberger W, Eckhof-Donovan S, Burdach S, Gobel U.

Thrombocytopenia and complement activation under recombinant TNF alpha/IFN gamma therapy in man. Annals of hematology. 1997;74:179-84.

[32] Simmons CP, Dong T, Chau NV, Dung NT, Chau TN, Thao le TT, et al. Early T-cell responses to dengue virus epitopes in Vietnamese adults with secondary dengue virus infections. Journal of virology.

2005;79:5665-75.

[33] Seneviratne SL, Malavige GN, de Silva HJ. Pathogenesis of liver involvement during dengue viral infections. Transactions of the Royal Society of Tropical Medicine and Hygiene. 2006;100:608-14. 
[34] Trung DT, Thao le TT, Hien TT, Hung NT, Vinh NN, Hien PT, et al. Liver involvement associated with dengue infection in adults in Vietnam. The American journal of tropical medicine and hygiene.

2010;83:774-80.

[35] Samanta J, Sharma V. Dengue and its effects on liver. World Journal of Clinical Cases : WJCC.

2015;3:125-31.

[36] Jagadishkumar K, Jain P, Manjunath VG, Umesh L. Hepatic Involvement in Dengue Fever in Children. Iranian Journal of Pediatrics. 2012;22:231-6.

[37] Roy A, Sarkar D, Chakraborty S, Chaudhuri J, Ghosh P, Chakraborty S. Profile of Hepatic Involvement by Dengue Virus in Dengue Infected Children. North American Journal of Medical Sciences. 2013;5:480-

5.

[38] Huang CM, Elin RJ, Ruddel M, Sliva C, Lotze MT, Rosenberg SA. Changes in laboratory results for cancer patients treated with interleukin-2. Clinical chemistry. 1990;36:431-4.

[39] Rathakrishnan A, Wang SM, Hu Y, Khan AM, Ponnampalavanar S, Lum LC, et al. Cytokine expression profile of dengue patients at different phases of illness. PloS one. 2012;7:e52215. 

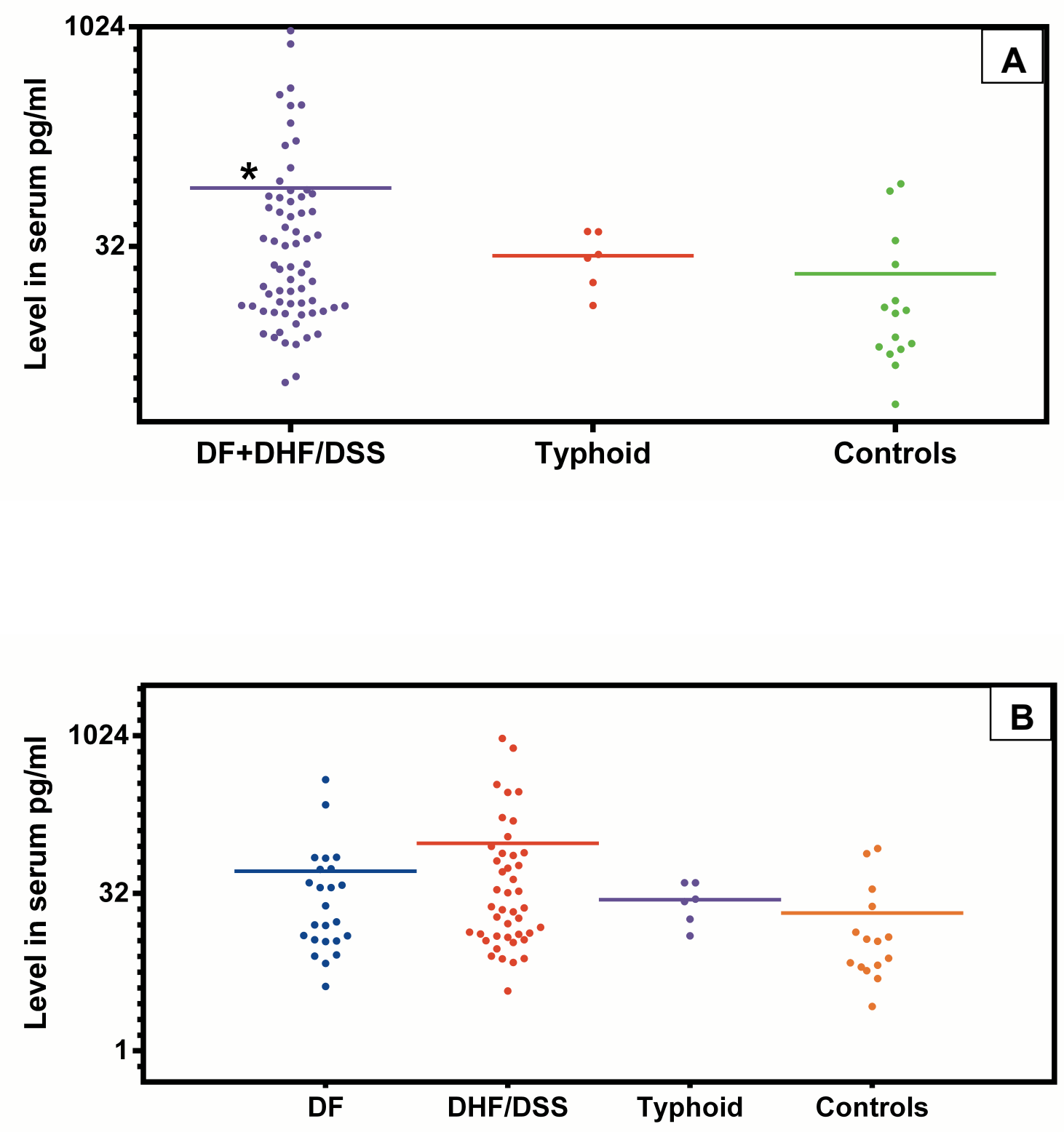

Figure 1A and 1B 

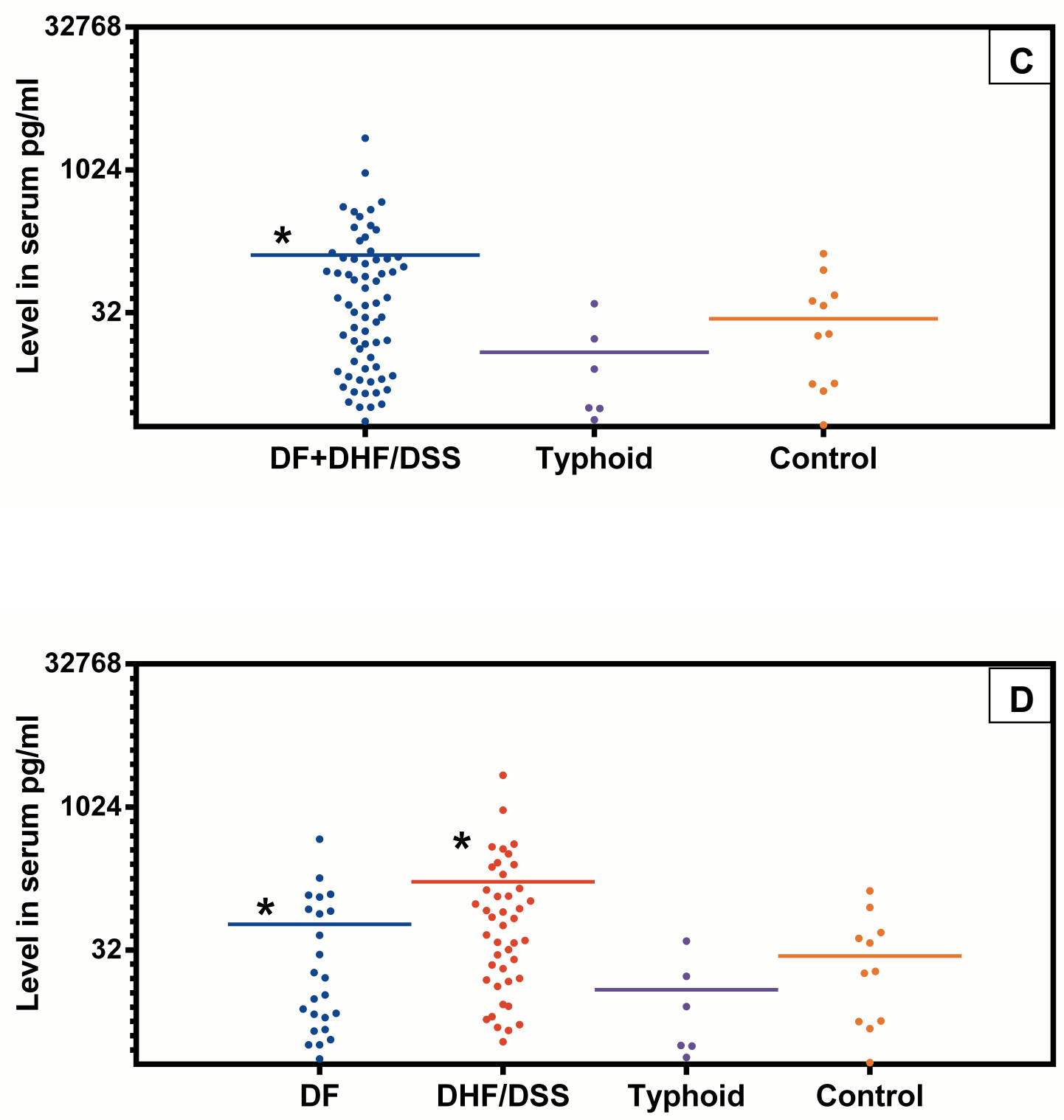

Figure 1C and 1D 


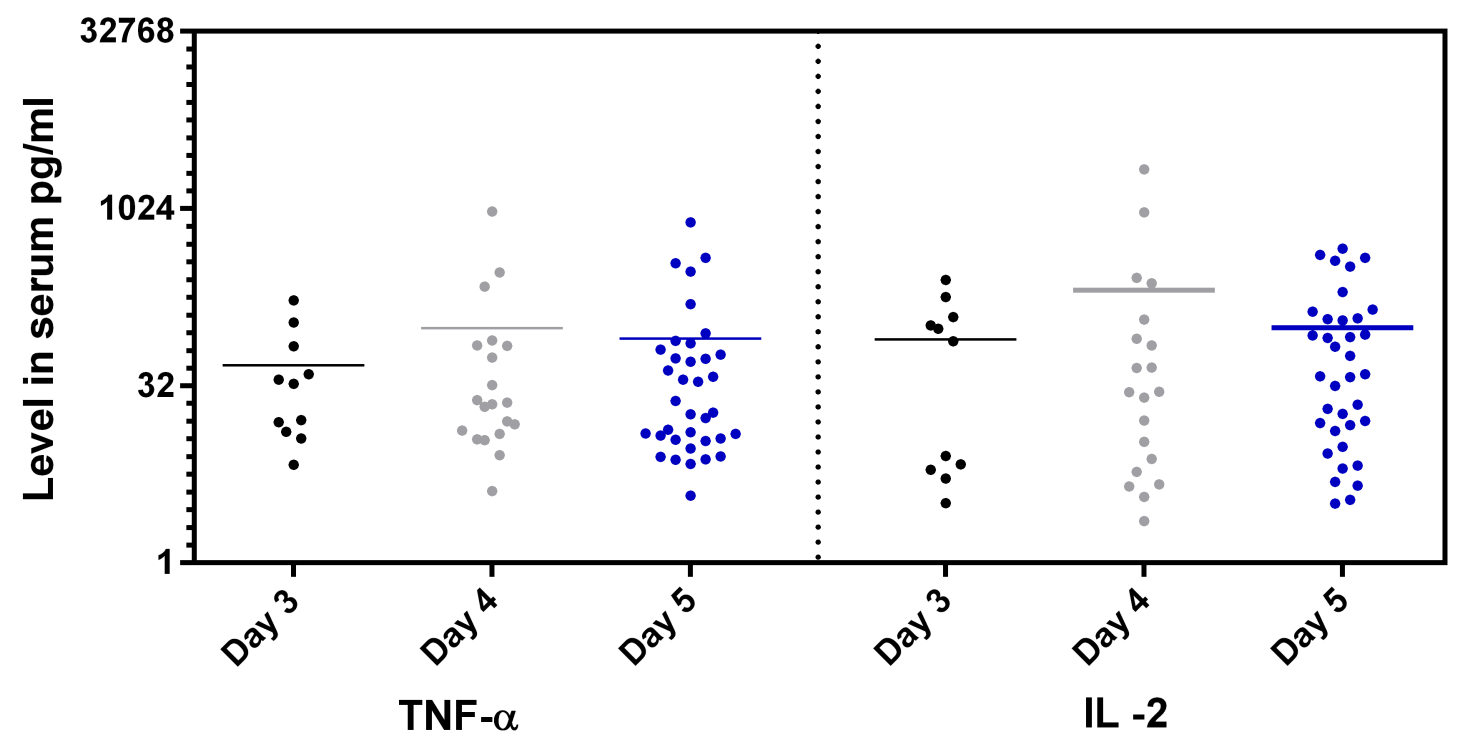

Figure 2 

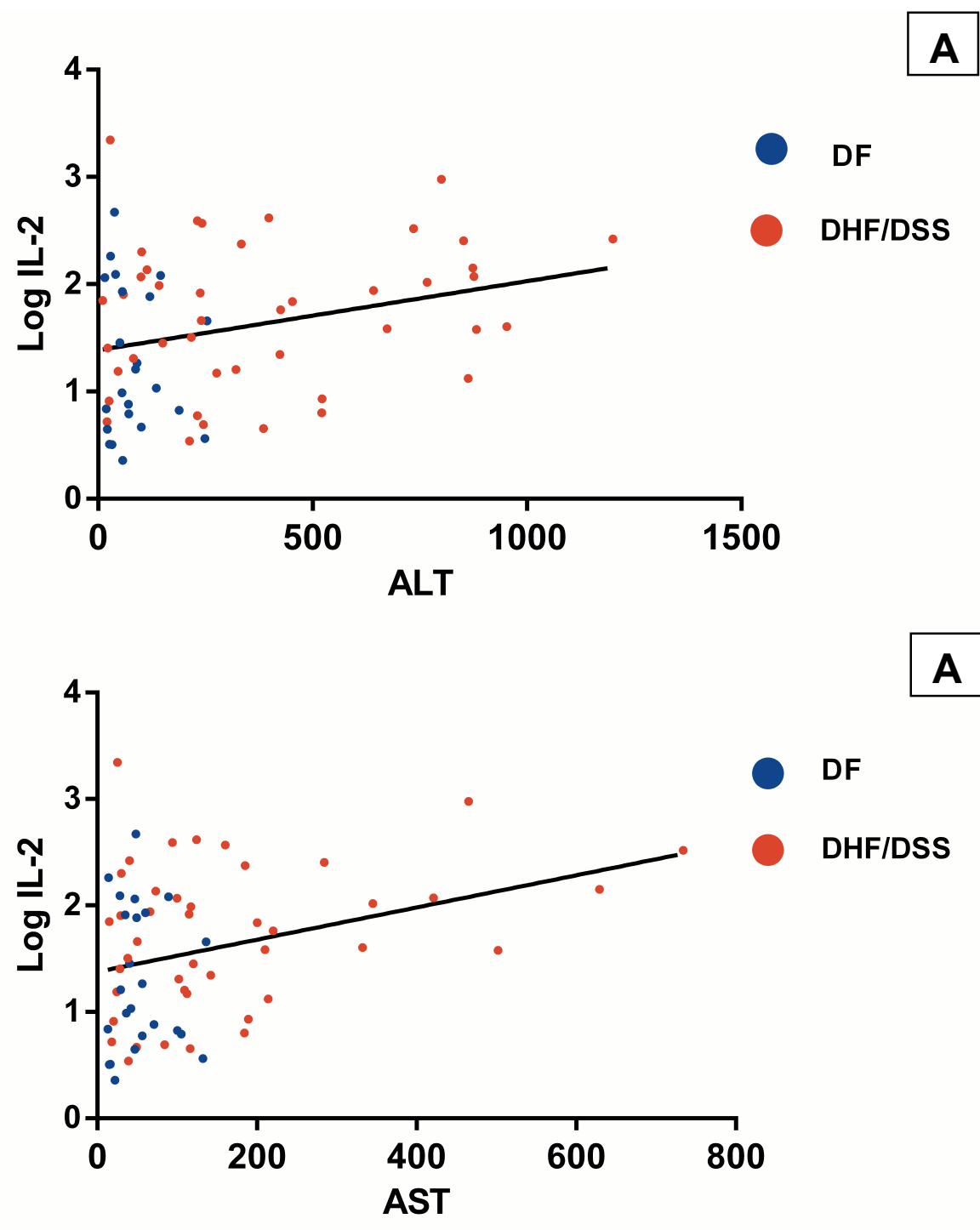

Figure 3 


\section{Tables}

2 Table 1. TNF- $\alpha$ and IL-2 levels in DF, DHF, typhoid patients and healthy afebrile controls.

\begin{tabular}{|c|c|c|c|c|c|}
\hline Category & $\begin{array}{l}\text { No of } \\
\text { cases }\end{array}$ & $\begin{array}{l}\text { TNF- } \alpha(p g / m l) \\
(\text { Mean } \pm \text { SD })\end{array}$ & $\begin{array}{l}\text { TNF- } \alpha \\
\text { (Range) }\end{array}$ & $\begin{array}{l}\text { IL-2 (pg/ml) } \\
(\text { Mean } \pm \text { SD) }\end{array}$ & $\begin{array}{l}\text { IL-2 } \\
\text { (Range) }\end{array}$ \\
\hline$\overline{\mathrm{DF}}$ & 24 & $51.78 \pm 17.37$ & $0-386.15$ & $59.68 \pm 101.05$ & $0-467.20$ \\
\hline DHF & 43 & $96.19 \pm 192.69$ & $0-961.58$ & $166.91 \pm 362.08$ & $0-2202.86$ \\
\hline Typhoid & 6 & $27.65 \pm 11.33$ & $0-27.86$ & $12.20 \pm 14.49$ & $0-37.21$ \\
\hline Controls & 15 & $20.72 \pm 25.98$ & $0-83.16$ & $27.49 \pm 38.76$ & $0-133.72$ \\
\hline
\end{tabular}

3

4

5 Table 2. Relationship between TNF- $\alpha$ and IL-2 levels and different laboratory parameters in the study sample ( $p$ 6 values as determined by Students unpaired $t$-test).

\begin{tabular}{lll}
\hline Laboratory parameter & TNF-a & IL-2 \\
\hline DENV RT-PCR positivity & 0.84 & 0.32 \\
PCV* & 0.72 & 0.39 \\
Leucopenia $\left(<5000 / \mathrm{mm}^{3}\right)$ & 0.03 & 0.24
\end{tabular}

Thrombocytopenia $\left(\leq 150,000 / \mathrm{mm}^{3}\right) \quad 0.68 \quad 0.57$

$\begin{array}{lcc}\text { Age } & 0.01 & 0.01 \\ \text { Primary DENV/Secondary DENV } & 0.13 & 0.24\end{array}$

\begin{tabular}{lcc} 
Time post fever & 0.69 & 0.37 \\
\hline IgM positivity & 0.49 & 0.39
\end{tabular}

*PCV- Packed cell volume.

8

9 
Table 3. Correlation values for the association of different laboratory parameters with TNF- $\alpha$ and IL-2 levels.

\begin{tabular}{|c|c|c|c|c|}
\hline \multirow[t]{2}{*}{ Comparison } & \multicolumn{2}{|c|}{ TNF- $\alpha$} & \multicolumn{2}{|c|}{ IL-2 } \\
\hline & p-value & Correlation & $p$-value & Correlation \\
\hline IL-2 & 0.59 & 0.07 & & \\
\hline WBC count on admission & 0.26 & -0.14 & $* * 0.02$ & 0.86 \\
\hline Platelet count & 0.80 & 0.03 & 0.86 & 0.02 \\
\hline Headache & 0.79 & -0.033 & 0.82 & 0.03 \\
\hline Retro orbital pain & 0.82 & -0.03 & 0.21 & 0.16 \\
\hline Myalgia & 0.44 & 0.10 & 0.08 & 0.22 \\
\hline Arthalgia & 0.48 & -0.09 & $* * 0.02$ & 0.28 \\
\hline Rash & 0.26 & 0.14 & 0.09 & 0.21 \\
\hline PTT* & 0.84 & -0.02 & 0.07 & 0.22 \\
\hline $\begin{array}{l}\text { Ratio of TNF- } \alpha \text { /IL- } 2 \text { in primary } \\
\text { infections }\end{array}$ & 0.32 & & 0.08 & \\
\hline $\begin{array}{l}\text { Ratio of TNF- } \alpha \text { / IL-2 in secondary } \\
\text { infections }\end{array}$ & 0.03 & & 0.87 & \\
\hline Difference in serotypes & 0.22 & 0.24 & 0.71 & -0.08 \\
\hline
\end{tabular}

13

14 *PTT- positive tourniquet test

15 indicates a strong correlation.

** A significant positive correlation between a laboratory or clinical parameter can only be interpreted when the $p$ value is $<0.05$ - For example when there is an increase in WBC there is also an increase in the IL-2 levels in the peripheral blood. The strength of the correlation is interpreted by the correlation value which when closer 1 